\title{
THERMODYNAMIC OPTIMIZATION OF AN IRREVERSIBLE REGENERATED BRAYTON HEAT ENGINE USING MODIFIED ECOLOGICAL CRITERIA
}

\author{
Ranjana Arora ${ }^{1, *}$, Rajesh Arora ${ }^{2}$
}

\begin{abstract}
The modified configuration of regenerated Brayton heat engine along with supplementary addition of heat in its irreversible mode is thermodynamically investigated and optimized. The definite temperature differential between system/reservoir is the source of external irreversibility and the losses because of rubbing/friction in turbine/compressor, regeneration heat losses and losses due to pressure drop are the internal irreversibilities considered in this analysis. The difference of output power and the exergy destruction rate, termed as ecological function, is thermodynamically optimized. It is found that regenerative effectiveness plays a vital role in obtaining maximum possible ecological function whereas output power and $1^{\text {st }}$ law efficiency predominantly depends on the cold side effectiveness in the system. It is also observed that the thermodynamic performance of proposed system/device is prominently depends on the efficiency of the turbine and consequently less dependent on compressor efficiency. The major outcome of this analysis is that with the inclusion of additional thermal heats at constant temperature conditions, various performance parameters i.e., output power (about 13\%) and $1^{\text {st }}$ law efficiency (about 9\%) of the model get improved significantly in comparison with the conventional gas power plant. Moreover, the model investigated in this study yields lesser output power, first law efficiency and ecological function and exactly follows the results/outcomes presented in the available literature at $\alpha_{1}=\alpha_{2}=1$, which are the pressure recovery coefficients at two ends.
\end{abstract}

Keywords: Modified Ecological Function, Irreversible Brayton Heat Engine, Regenerators, Isothermal Heat Addition, Thermodynamic Optimization

\section{INTRODUCTION}

The energy conversion processes based on Brayton cycles have got wide range of utilities in numerous fields viz. gas power stations, ship propulsion, air crafts and other industrial mechanisms. The differential outcomes of output power and exergy destruction rate is proposed as ecological function [1] and they computed the corresponding first law efficiency of endoreversible mode operated Carnot heat engine which is the mean of Curzon-Ahlborn/ Carnot efficiencies. Further, Yan [2] modified Angulo-Brown's ecological [1] function by replacing sink temperature with environment temperature as sink-side temperature is not the same as of the ambient one. Veccguarelli et al. [3] examined the efficiency of Brayton heat engine along with dual thermal addition and observed the increased efficiency with the application of heat inclusion at constant temperature conditions. Cheng and Chen ecologically optimized Brayton heat engine on the basis of endoreversible [4] and irreversible [5] configurations and observed significant decrease in rate of entropy generation for trivial fall in output power. Goktun and Yavuz [6] investigated the performance of gas turbine engine with two heat additions and found appreciable enhancement in first law efficiency of about 10\% compared with conventional heat engines. They also observed that gas turbine engines at $\mathrm{r}_{\mathrm{p}}<12$, gives good results with the use of regenerator. Erbay et al. [7] analyzed Brayton cycle and proposed optimal design for gas turbines/engines for isothermal heat inclusion. Kaushik and Tyagi [8] employed finite time thermodynamic (FTT) principles on regenerative Brayton heat engine in irreversible mode. Later, Arora et al. [9] employed multiobjective thermoeconomic principles on the regenerative Braytone cycle in order to calculate the optimum values of several design/input parameters. Ust et al. [10-11] performed optimization based on ecological function for an endoreversible mode of Brayton cycle. Later Arora et al. [12-15] performed various thermodynamic and ecological optimization studies for Stirling/Ericsson cycles and figured out the optimal points of various input/design variables for these cycles. Further, Kaushik et al. [16] carried out thermodynamic investigation on regenerated Brayton cycle in an 
irreversible mode along with heat addition at constant temperature. They optimized output power in context with operating temperatures and observed a significant enhancement of about $15 \%$ in first law efficiency of the system. In addition to this, Tyagi et al. [17] examined a Brayton heat engine for maximizing ecological function and computed the optimal values of different operating/output parameters for which system attains its highest possible values of first law efficiency, output power and the ecological function. Kumar et al. [18] analyzed an irreversible Brayton system in context with the ecological criterion. They found that regenerative effectiveness is more prominent for the maximization of ecological function and its respective first law efficiency whereas sink/cold zone effectiveness for output power of the system. In due time, various ecological function optimizations are found in the current literature for the proposed system in endo-and irreversible mode [19-28]. Razmara [29] employed exergy-based approach to internal combustion engines in order to improve its thermal efficiency. Later, Hajmohammadi et al. [30-33] carried out various heat transfer studies in order to provide the optimal design of different types of fins. The present work is an extension of work done on Brayton cycle by incorporating isothermal heat addition in the proposed model and output power and $1^{\text {st }}$ law efficiency is formulated at the maximum ecological function, and regenerated Brayton heat engine in irreversible mode. The thermodynamic impacts of different heat exchanger effectiveness, turbine/compressor efficiency, rate of heat capacitance rates, pressure dip ratios at constant temperature values and pressure recovery coefficients are investigated and formulated. Moreover, the thermodynamic model of Brayton heat engine investigated here yields superior values of first law efficiency, output power and ecological function which is true while considering the parametric values of addition heat addition in the current system. The present work can further be extended by applying various evolutionary algorithm techniques [34-48] in order to get optimum design of regenerated Brayton heat engine with constant heat addition.

\section{STATE OF ART}

In the present work, the Brayton cycle with constant temperature heat addition has been chosen and thermodynamic model is developed using FTT principles. Certain assumptions are made in developing the model which are described as follows:

(a) The steady state operation of the proposed system is assumed.

(b) The heat source/sink have finite/fixed heat capacity.

(c) The external irreversibility because of finite temperature differential whereas internal irreversibility due to turbine/compressor isentropic efficiencies, regenerated heat losses and pressure drops are considered.

(d) The constant specific heat of the working medium is undertaken.

(e) The working medium is supposed to act as an ideal gas.

The modified ecological function, i.e., difference of output power and the exergy destruction rate, is chosen as an objective for optimization. Afterwards, the comprehensive performance evaluation and optimization has been accomplished with the view of optimizing power output/thermal efficiency of the system with respect of working medium temperatures. The developed model is validated by comparing the outcomes with the previous literature. Moreover, the impact of isothermal heat addition on the system performance/output is observed.

\section{DESCRIPTION OF THE MODEL}

Fig. 1 (a) shows the regenerative Brayton heat engine in irreversible mode equipped with heat source/sink of definite thermal dimensions. In this system, state 1 and 2 shows the entry nodes and compressed zone of the working media, which afterwards get into a regenerator. It is then heated upto point $2 \mathrm{R}$, with the help of a turbine exhaust. After this, the fluid gets along a heat exchanger with a certain pressure fall as indicated by pressure recovery coefficient, $\alpha_{2}=\mathrm{p}_{1} / \mathrm{p}_{5}$ and heated upto a point state 3 . In step 1 , heat inclusion in the regular combustion chamber (RCC) takes place at same pressure along hot zone of heat exchangers. Conversely, the temperature of the source goes down from $\mathrm{T}_{\mathrm{H} 1}$ to $\mathrm{T}_{\mathrm{H} 2}$. In step 2, inclusion of thermal heat $\mathrm{Q}_{\mathrm{H} 1}$ takes place in the Converging Combustion Chamber (CCC) at fixed temperature condition in process 3 to 4 as indicated in Fig. 1 (a). Due to this, there occurs the fall in temperature for the heat source from $\mathrm{T}_{\mathrm{H} 3}$ to $\mathrm{T}_{\mathrm{H} 4}$. Thereafter, the fluids get into the regenerator to shed some of the thermal energy and then to cold/sink side heat exchanger along a slight pressure drop shown by another pressure recovery coefficient, $\alpha_{2}=\mathrm{p}_{1} / \mathrm{p}_{5}$. 


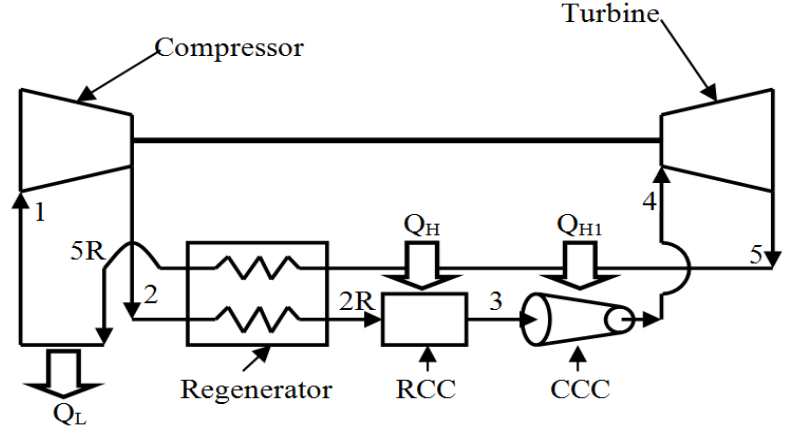

Figure 1(a). Brayton heat engine with converging combustion chamber

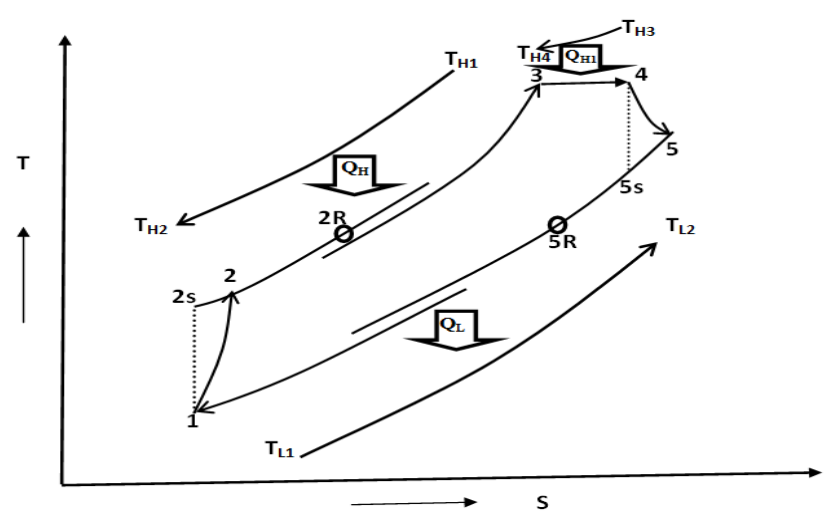

Figure 1(b). Temperature-entropy/TS diagram for Brayton heat engine with regeneration

The working media encounters a drop-in temperature till it reaches state 1 , on the other hand the temperature of the sink rises from $T_{\mathrm{L} 1}$ to $\mathrm{T}_{\mathrm{L} 2}$. Henceforth, the whole process i.e., (1-2-2R-3-4-5-5R-1) along compressed/expanded state with drop in pressure irreversibility for a definite thermal capacity of outer reservoirs. The subsequent processes (1-2s) and (4-5s) are constant entropy in behavior as illustrated by spotted outlines in Fig. 1(b).

\section{THERMODYNAMIC ANALYSIS}

The different heat transfers are evaluated as:

$$
\begin{gathered}
Q_{H}=U_{H} A_{H} \lambda_{H}=C_{H}\left(T_{H 1}-T_{H 2}\right) \\
Q_{H 1}=U_{H 1} A_{H 1} \lambda_{H 1}=C_{H 1}\left(T_{H 3}-T_{H 4}\right) \\
Q_{L}=U_{L} A_{L} \lambda_{L}=C_{L}\left(T_{L 2}-T_{L 1}\right) \\
Q_{R}=U_{R} A_{R} \lambda_{R}=C_{W}\left(T_{2 R}-T_{2}\right)
\end{gathered}
$$

where,

$$
\lambda_{H}=\frac{\left(T_{H 1}-T_{3}\right)-\left(T_{H 2}-T_{2 R}\right)}{\ln \left\{\left(T_{H 1}-T_{3}\right) /\left(T_{H 2}-T_{2 R}\right)\right\}}
$$




$$
\begin{aligned}
& \lambda_{H 1}=\frac{\left(T_{H 3}-T_{3}\right)-\left(T_{H 4}-T_{3}\right)}{\ln \left\{\left(T_{H 3}-T_{3}\right) /\left(T_{H 4}-T_{3}\right)\right\}} \\
& \lambda_{L}=\frac{\left(T_{5 R}-T_{L 2}\right)-\left(T_{1}-T_{L 1}\right)}{\ln \left\{\left(T_{5 R}-T_{L 2}\right) /\left(T_{1}-T_{L 1}\right)\right\}} \\
& \lambda_{R}=\frac{\left(T_{5}-T_{2 R}\right)-\left(T_{5 R}-T_{2}\right)}{\ln \left\{\left(T_{5}-T_{2 R}\right) /\left(T_{5 R}-T_{2}\right)\right\}}
\end{aligned}
$$

Using equations (1) to (8),

$$
\begin{gathered}
Q_{H}=\varepsilon_{H} C_{H, \min }\left(T_{H 1}-T_{2 R}\right)=C_{W}\left(T_{3}-T_{2 R}\right) \\
Q_{H 1}=\varepsilon_{H 1} C_{H 1, \text { min }}\left(T_{H 3}-T_{3}\right) \\
Q_{L}=\varepsilon_{L} C_{L, \min }\left(T_{5 R}-T_{L 1}\right)=C_{W}\left(T_{5 R}-T_{1}\right) \\
Q_{R}=\varepsilon_{R} C_{W}\left(T_{5}-T_{2}\right)=C_{W}\left(T_{5}-T_{5 R}\right)
\end{gathered}
$$

Here $\varepsilon_{\mathrm{H}}, \varepsilon_{\mathrm{H} 1}, \varepsilon_{\mathrm{L}} / \varepsilon_{\mathrm{R}}$ are the effectivenesses of the constant pressure heat source zone, constant temperature thermal source zone, sink/regenerated heat exchanger respectively.

Further, the component efficiencies of turbine/compressor are written as:

$$
\begin{gathered}
\eta_{\text {comp }}=\frac{T_{2 s}-T_{1}}{T_{2}-T_{1}} \\
\eta_{\text {turb }}=\frac{T_{4}-T_{5}}{T_{4}-T_{5 s}}
\end{gathered}
$$

Solving Eqns (7-14), gives,

$$
\begin{gathered}
T_{5 \mathrm{R}}=\left(1-\varepsilon_{\mathrm{R}}\right) T_{5}+\varepsilon_{\mathrm{R}} T_{2} \\
T_{2 \mathrm{R}}=\left(1-\varepsilon_{\mathrm{R}}\right) T_{2}+\varepsilon_{\mathrm{R}} T_{5} \\
T_{1}=(1-y) T_{5 \mathrm{R}}+y T_{L 1} \\
T_{3}=(1-x) T_{2 \mathrm{R}}+x T_{H 1} \\
T_{2 s}=\left(1-\eta_{\text {comp }}\right) T_{1}+T_{2} \eta_{\text {comp }} \\
T_{5 s}=\left(1-\eta_{\text {turb }}^{-1}\right) T_{3}+T_{5} \eta_{\text {turb }}^{-1}
\end{gathered}
$$

Apply second law of thermodynamics for an irreversible regenerative Brayton model, 


$$
T_{1} T_{3}=\beta T_{2 s} T_{5 s}
$$

Here, $\beta=\left(\chi_{t} \alpha_{1} \alpha_{2}\right)^{\frac{k-1}{k}}$ and $\chi_{t}$ is the constant temperature pressure drop ratio, i.e., $\mathrm{p}_{4} / \mathrm{p}_{3}$. Putting the values of various temperatures in Eqn. (21), one can get,

$$
X_{1} T_{2}^{2}+X_{2} T_{2}+X_{3}=0
$$

The values of $\mathrm{P}, \mathrm{Q}$ and R, are recorded in the Appendix. Solution of equation (22) is obtained as:

$$
T_{2}=\frac{-X_{2}+\sqrt{X_{2}^{2}-4 X_{1} X_{3}}}{2 X_{1}}
$$

Applying $1^{\text {st }}$ law of thermodynamics,

$$
P_{B h e}=Q_{H}+Q_{H 1}-Q_{L}=\varepsilon_{H} C_{H, \text { min }}\left(T_{H 1}-T_{2 R}\right)+\varepsilon_{H 1} C_{H 1, \text { min }}\left(T_{H 3}-T_{3}\right)-\varepsilon_{L} C_{L, \text { min }}\left(T_{5 R}-T_{L 1}\right)
$$

Again, substituting equations (15-20), into equations (24), P can be written as:

$$
\begin{gathered}
P_{\text {Bhe }}=z_{6}-x_{7} T_{2}-y_{7} T_{5} \\
\eta_{I}=\frac{P_{\text {Bhe }}}{Q_{H}+Q_{H 1}}=\frac{z_{6}-x_{7} T_{2}-y_{7} T_{5}}{z_{7}-x_{8} T_{2}-y_{8} T_{5}}
\end{gathered}
$$

The values of different parameters are recorded in the Appendix.

The second law/exergy efficiency is given as

$$
\eta_{I I}=\frac{\eta_{I}}{\eta_{\text {rev }}}
$$

where,

$$
\eta_{\text {rev }}=1-\frac{T_{L}}{T_{H}}
$$

The modified ecological function chosen for optimization as proposed by refs. [1-2] is

$$
E=P_{B h e}-E x_{D}
$$

Here $\mathrm{T}_{0}$ is the environment temperature, $\mathrm{Ex}_{\mathrm{D}}$ is the exergy destruction rate.

$$
\begin{aligned}
& E=P_{B h e}-T_{0}\left(\frac{Q_{L}}{T_{L 1}}-\frac{Q_{H}}{T_{H 1}}-\frac{Q_{H 1}}{T_{H 3}}\right) \\
& E=z_{9}-x_{10} T_{2}-y_{10} T_{4}
\end{aligned}
$$

Parameters $\mathrm{z}_{9}, \mathrm{x}_{10}$ and $\mathrm{y}_{10}$ are given in nomenclature. 
Therefore, Eqn. (30) can be optimized with respect to $\mathrm{T}_{5}$ i.e. $\frac{\partial E}{\partial T_{5}}=0$ and quadratic equation in $\mathrm{T} 5$ is obtained as:

$$
Y_{1} T_{5}^{2}+Y_{2} T_{5}+Y_{3}=0
$$

Parameters $\mathrm{Y}_{1}, \mathrm{Y}_{2}$ and $\mathrm{Y}_{3}$ are noted in the Appendix.

Resolving equation (31), one can get,

$$
T_{5, \text { opt }}=\frac{-Y_{2}-\sqrt{Y_{2}^{2}-4 Y_{1} Y_{3}}}{2 Y_{1}}
$$

\section{RESULTS AND DISCUSSION}

The obtained results in numerically appreciated form are explored while analyzing the impacts of different output/performance parameters viz. turbine/compressor efficiency, effectivenesses of different heat exchangers, pressure dip at constant temperature, recovery coefficients at drop in pressure and rate of heat capacitances of the working media on regenerated Brayton cycle in irreversible mode. The influence of aforesaid factors are analyzed while considering the remaining parameters as the constant values [8] as given $\mathrm{T}_{\mathrm{H} 3}=1250 \mathrm{~K}$, $\mathrm{T}_{\mathrm{H} 1}=1000, \mathrm{~T}_{\mathrm{L} 1}=300 \mathrm{~K}, \mathrm{~T}_{0}=295 \mathrm{~K}, \eta_{\text {turb }}=\eta_{\text {comp }}=0.8, \mathrm{C}_{\mathrm{W}}=1.05 \mathrm{kWK}^{-1}, \mathrm{C}_{\mathrm{H}}=\mathrm{C}_{\mathrm{H} 1}=\mathrm{C}_{\mathrm{L}}=1 \quad \mathrm{kWK}^{-1}, \mathrm{U}_{\mathrm{H}}=$ $\mathrm{U}_{\mathrm{H} 1}=\mathrm{U}_{\mathrm{L}}=\mathrm{U}_{\mathrm{R}}=2.0 \mathrm{kWK}^{-1} \mathrm{~m}^{-2}, \chi_{\mathrm{t}}=0.8, \alpha_{1}=\alpha_{2}=0.95$. The detailed discussion of the obtained outcomes is discussed in the subsequent sections.

Impacts of $\varepsilon_{\mathrm{H} 1}, \varepsilon_{\mathrm{H}}, \varepsilon_{\mathrm{R}}$ and $\varepsilon_{\mathrm{L}}$

The thermodynamic influence of different effectivenesses are presented in Figs. 2 (a-c) on the output power, $1^{\text {st }}$ law efficiency and the objective/ecological function. It is well notified from the obtained results that the highest attainable values of output power, $1^{\text {st }}$ law efficiency and objective function rises as the constant temperature heat sink/source side effectivenesses and regenerated heat exchanger effectiveness are raised. On the other hand, as constant preheat source side effectiveness, goes up, all performance factors suffers slight fall in the system. It is also observed that the influence of $\left(\varepsilon_{\mathrm{L}}\right)$ is much more dominant on first law efficiency and output power whereas ecological function primarily depends on $\left(\varepsilon_{R}\right)$. The obtained outcomes may be shown their relations with heat exchanger area of the engine, which needs to be as maximum as possible for obtaining maximum effectiveness. Eventually, the cost of the system goes up with the heat transfer area. Therefore, one has to be vigilant while choosing the effectiveness factor for different heat exchanger designs. Generally, the disparities of several performance constraints in context with this factor are not its direct function and $\varepsilon_{\mathrm{L}}>\varepsilon_{\mathrm{H} 1}>$ $\varepsilon_{\mathrm{H}}$ is the best and optimized relation for realizing the practical systems.

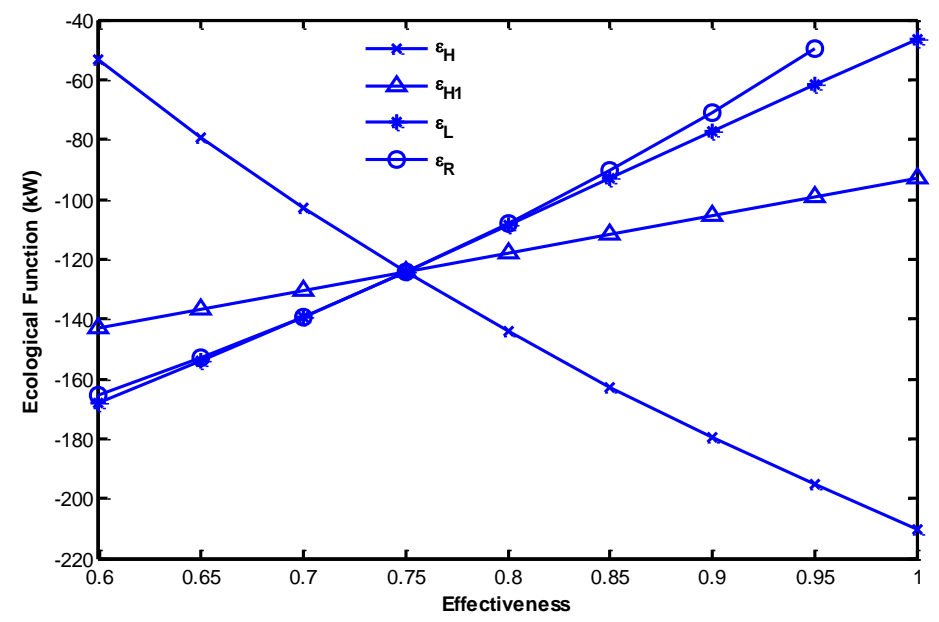

Figure 2(a). Impacts of different effectivenesses on ecological function 


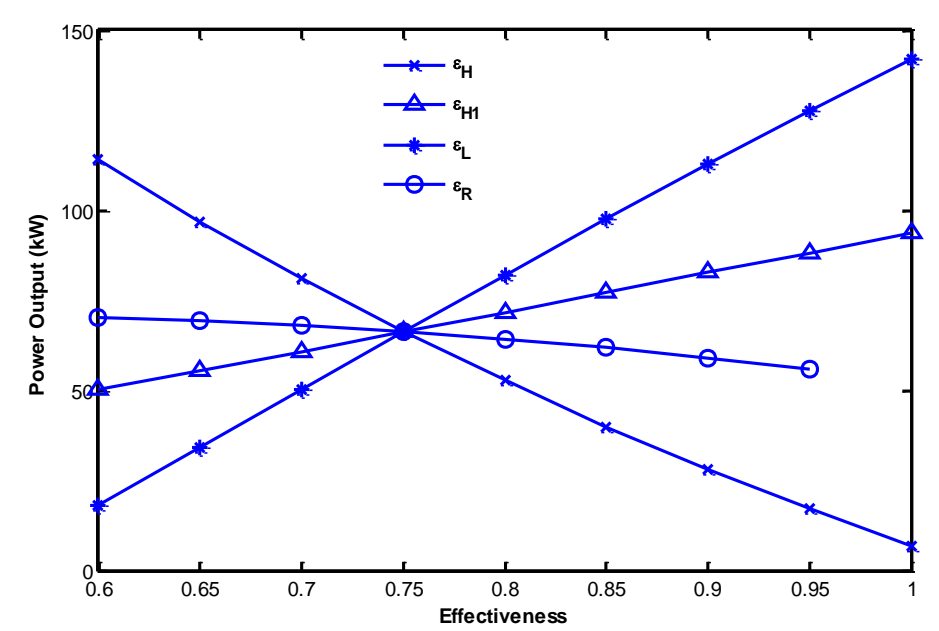

Figure 2(b). Effects of different effectivenesses on the output power

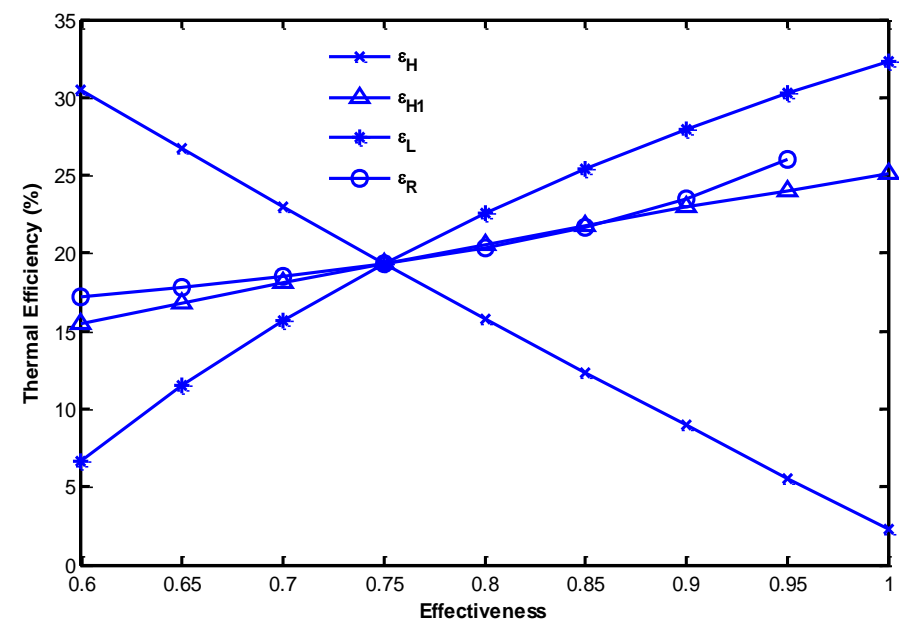

Figure 2(c). Effects of different effectivenesses on the thermal efficiency

\section{Impacts of $\mathrm{C}_{\mathrm{H}}, \mathrm{C}_{\mathrm{H} 1}, \mathrm{C}_{\mathrm{L}}$ and $\mathrm{C}_{\mathrm{W}}$}

Figs. 3 (a-c) shows the influence of different thermal capacitance rates on highest possible values of $1^{\text {st }}$ law efficiency, output power, and ecological function. It is very much clear that output power/ $1{ }^{\text {st }}$ law efficiency rises with the rise in rate of heat capacitance for fixed temperature source/ sink side reservoir and cycle working fluid whereas all the performance parameters reveal sharp dip along the rise in rate of heat capacitance at constant pressure thermal source reservoir. It is also observed, the rate of heat capacitance along sink side is more prominent on the performance factors compared to fixed temperature source zone of the system model. Generally, the change in different performance factors for the rate of heat capacitance does not result in liner relationship and the mutual relation $\mathrm{C}_{\mathrm{L}}>\mathrm{C}_{\mathrm{H} 1}>\mathrm{C}_{\mathrm{W}}$ is found to be best suited for system execution in most optimized manner. The rate of heat capacitance at fixed pressure thermal reservoir must be as small as possible.

\section{Impacts of turbine/compressor efficiencies}

The change of compressor/turbine efficiencies on max. ecological/output power and $1^{\text {st }}$ law efficiency of regenerative Brayton cycle in irreversible mode along definite capacity thermal reservoir are illustrated in Fig. 4 (a-c). It is found here that the highest possible objective function, output power and first law efficiency rises with the rise in component efficiencies which shows that the higher the values of component efficiencies are, better the system performance is achieved. It is also observed that the impact of turbine efficiency is dominant on the thermodynamic system outcome compared with the compressor efficiency. Consequently, for the designing of practical Brayton cycle systems, exhaustive literature search and investigation is mandatory on the efficiency of compressor. 


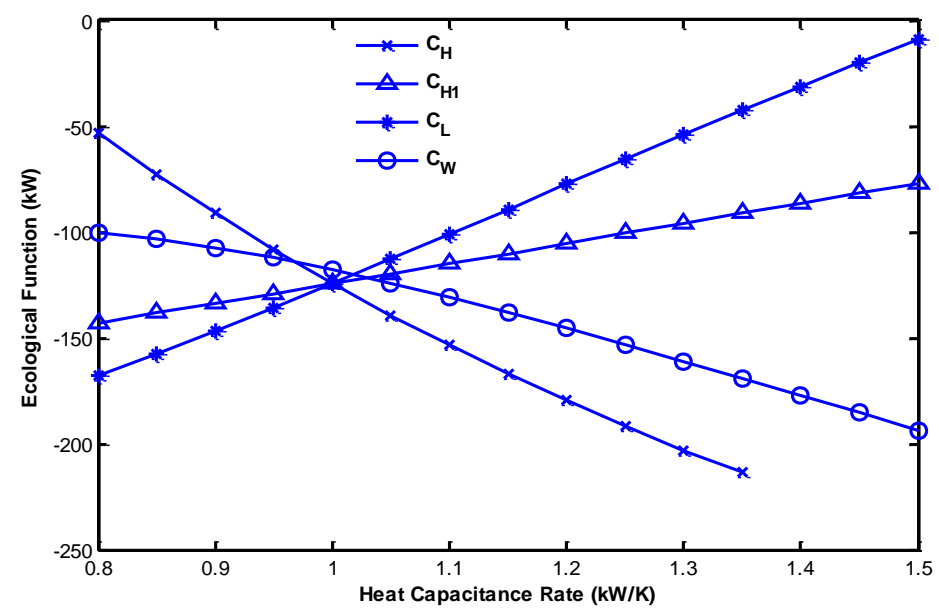

Figure 3(a). Impacts of various heat capacitances on the objective/ecological function

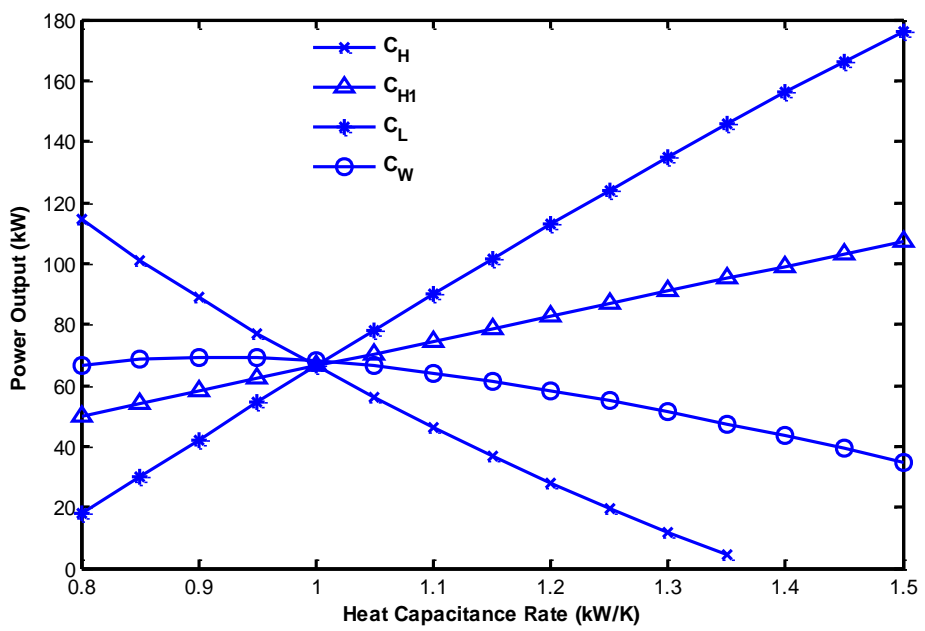

Figure 3(b). Impacts of different heat capacitances on the power output

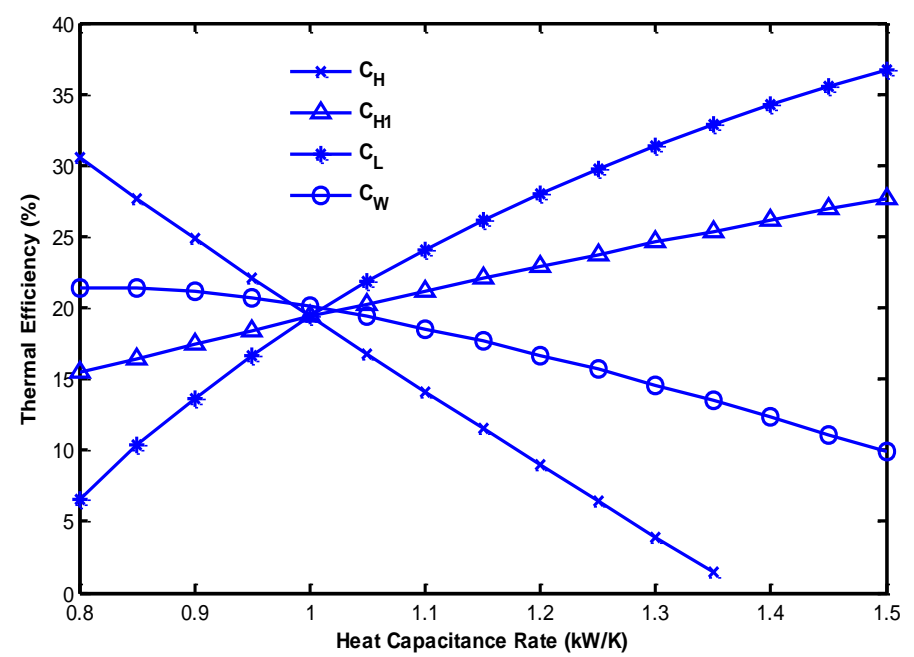

Figure 3(c). Effects of different heat capacitance rates on the thermal efficiency 


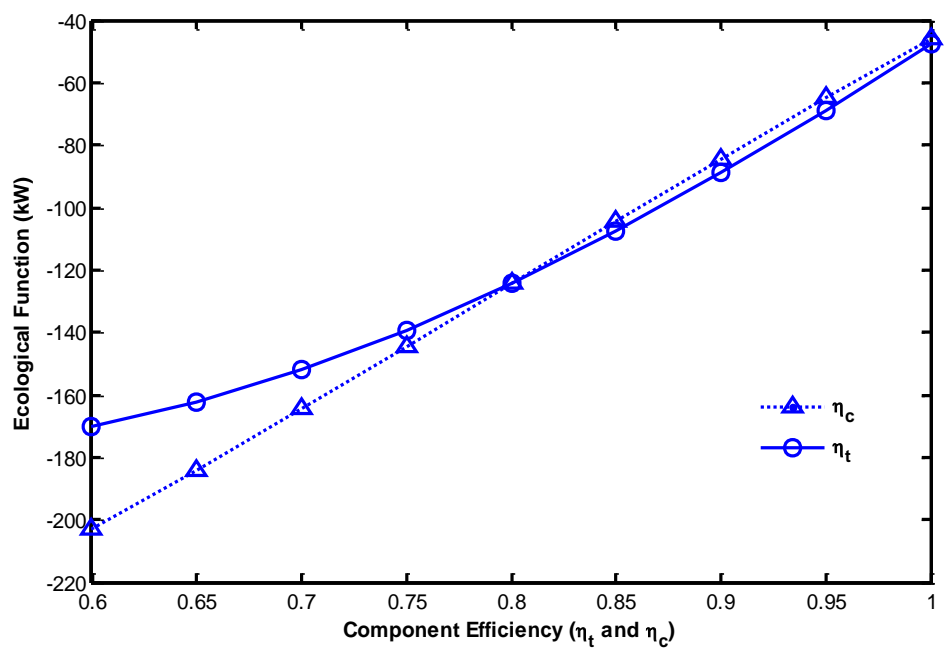

Figure 4(a). Effects of different component efficiencies on the ecological function

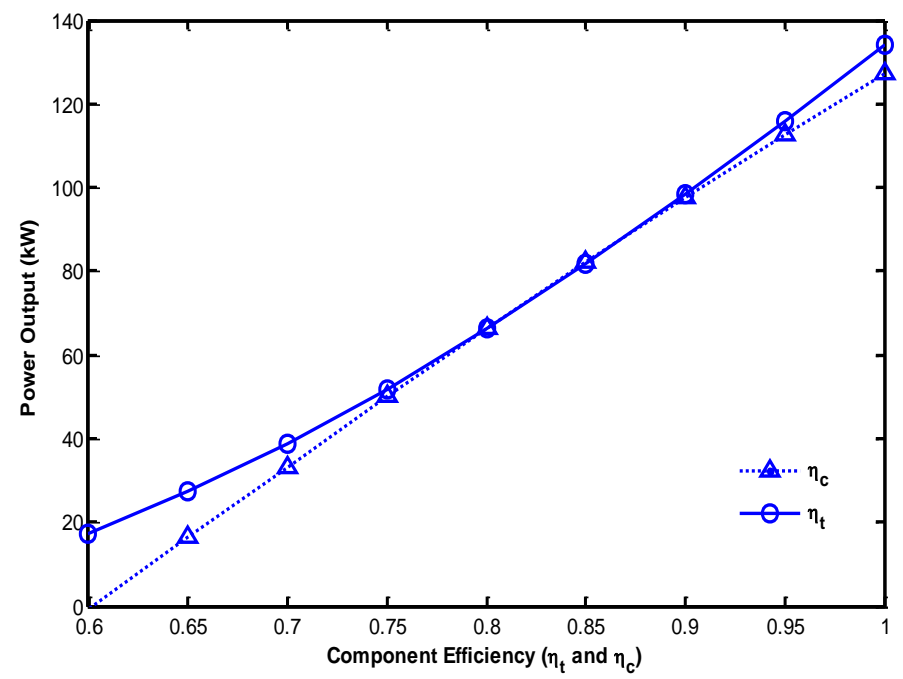

Figure 4(b). Effects of different component efficiencies on the output power

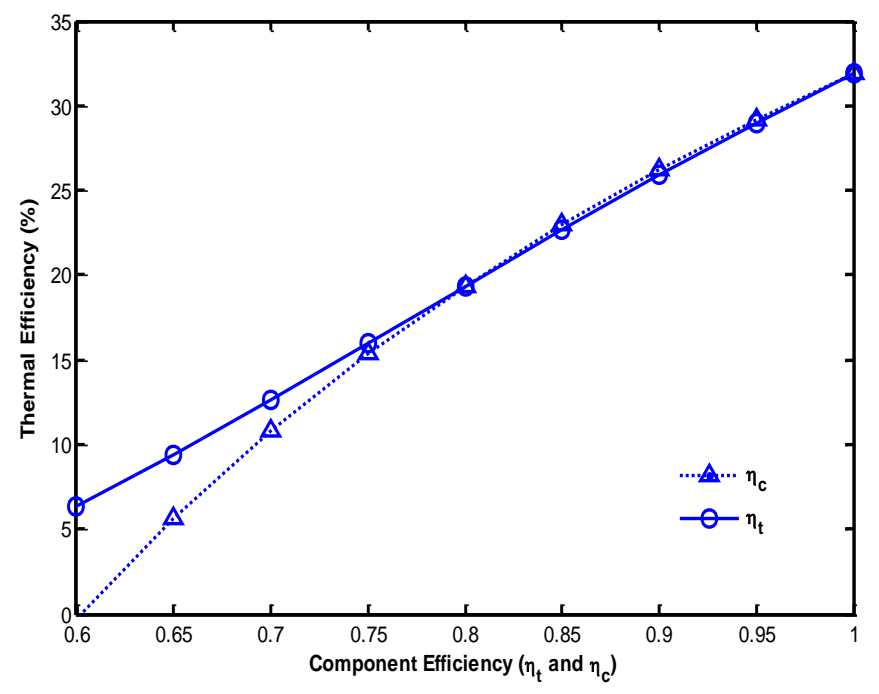

Figure 4(c). Impacts of different component efficiencies on the thermal efficiency

\section{Impacts of pressure recovery coefficient}

Fig. 5 illustrates the influence of pressure recovery coefficient on different outcome factors of Brayton cycle in irreversible mode. It has been observed that highest ecological/output power and first law efficiency goes 
up as the pressure drop falls. It is also observed that the different performance factors achieve their highest values at zero magnitude of pressure drop which is nearly impossible to attain in practical Brayton system. Furthermore, highest ecological function, output power and first law efficiency possess linear variations with respect to pressure recovery coefficients.

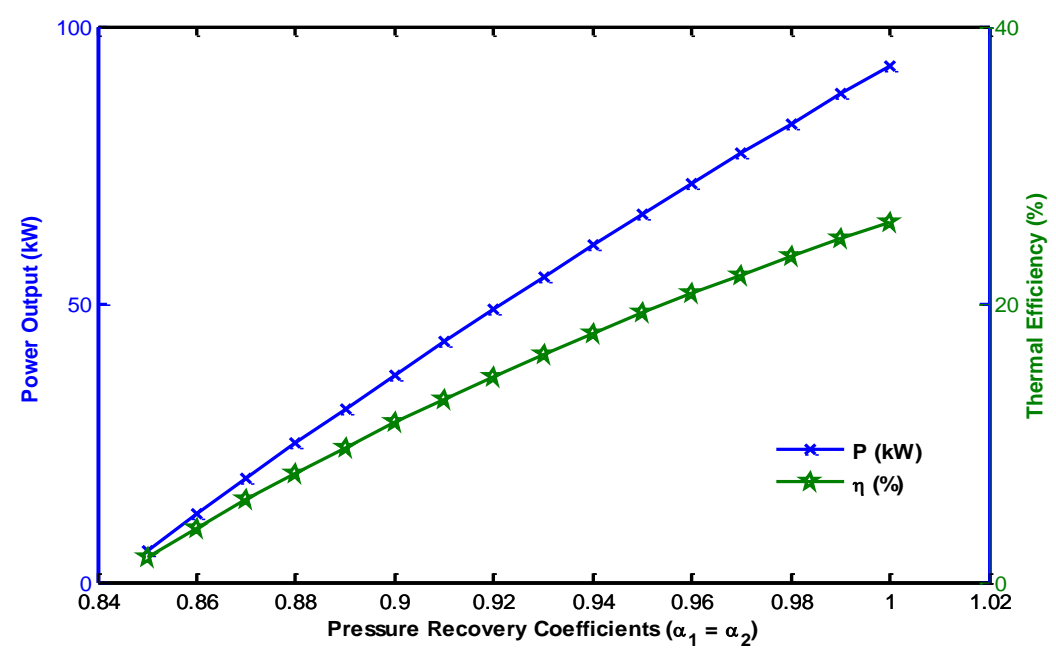

Figure 5. Effects of pressure recovery coefficients on output power and thermal efficiency

\section{Impacts of isothermal pressure drop ratio $\left(p_{4} / p_{3}\right)$}

Fig. 6 reveals the influence of fixed temperature pressure ratio $(\mathrm{p} 4 / \mathrm{p} 3)$ on different output factors of Bratyon system in irreversible mode. It is found that the highest ecological function, output power and first law efficiency goes up as this ratio (p4/p3) rises in the system. Subsequently, different output parameters achieve their highest possible value as this ratio attains unity magnitude, which is nearly impossible to be attined in real Bratyon heat engine cycles. Afterwards, highest ecological function, output power and first law efficiency possess linear relationship with the fixed temperature pressure ratio $(\mathrm{p} 4 / \mathrm{p} 3)$.

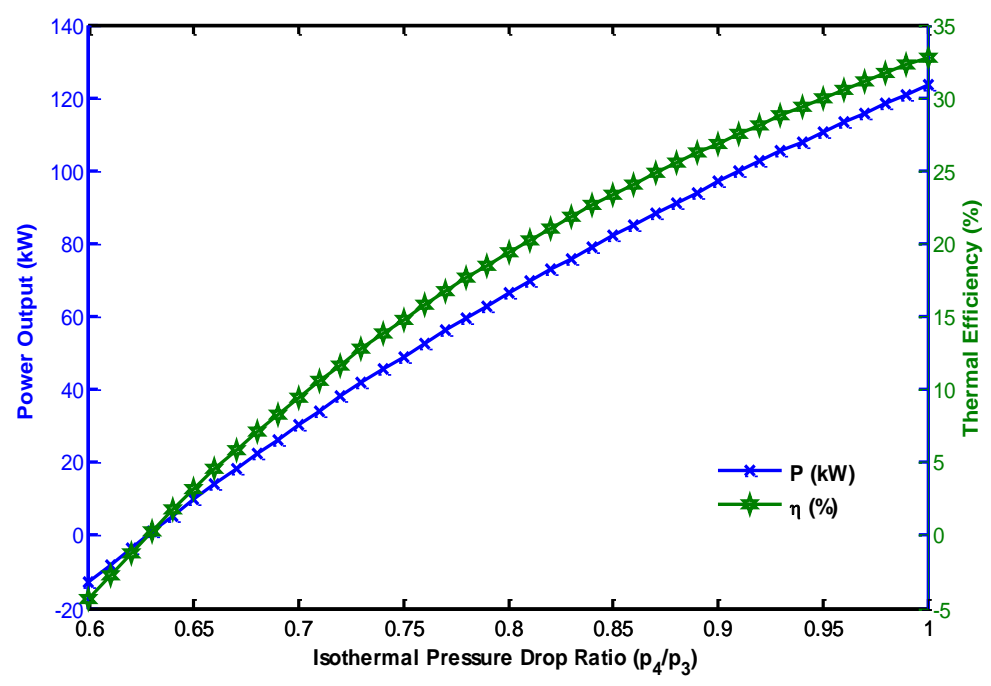

Figure 6. Impacts of pressure drop ratio on output power/ $1^{\text {st }}$ law efficiency

\section{Comparison of outcomes with previous literature}

The comparison of outcomes/results attained for an irreversible Brayton cycle has been done with the previous literature [8, 16-17] as illustrated in Table 1 . It has been noticed that the results of output power/ $1^{\text {st }}$ law efficiency are in coherence with the available literature. It proves the correctness of the developed model. Furthermore, the proposed model exactly follows the results/outcomes presented in the available literature at $\alpha_{1}=\alpha_{2}=1$, which are the pressure recovery coefficients at two ends. 
Table 1. Comparison of outcomes with previous literature

\begin{tabular}{|l|c|c|c|c|c|c|c|}
\hline \multirow{2}{*}{ Study } & \multicolumn{6}{|c|}{ Input parameters } & \multicolumn{2}{c|}{ Output parameters } \\
\cline { 2 - 9 } & $\boldsymbol{\varepsilon}_{\mathbf{H}}$ & $\boldsymbol{\varepsilon}_{\mathbf{H} 1}$ & $\boldsymbol{\varepsilon}_{\mathbf{L}}$ & $\boldsymbol{\varepsilon}_{\mathbf{R}}$ & $\mathbf{T}_{\mathbf{5}}$ & $\mathbf{P}(\mathbf{k W})$ & $\boldsymbol{\eta}$ \\
\hline $\begin{array}{l}\text { Present study } \\
\text { (With Isothermal Heat Addition) }\end{array}$ & 0.81 & 0.8 & 0.8 & 0.8 & 958.62 & 68.82 & 0.1986 \\
\hline $\begin{array}{l}\text { Present study } \\
\text { (Without Isothermal Heat Addition) }\end{array}$ & 0.81 & - & 0.8 & 0.8 & 932.35 & 61.25 & 0.1829 \\
\hline Ref [8] & 0.75 & 0.75 & 0.75 & 0.75 & - & 63 & 0.1858 \\
\hline Ref [16] & 0.75 & 0.75 & 0.75 & 0.75 & - & 110 & 0.27 \\
\hline Ref [17] & 0.8 & 0.8 & 0.8 & 0.8 & - & 112 & 0.26 \\
\hline
\end{tabular}

\section{CONCLUSION}

The Brayton heat engine possessing design/performance parameters near to the real one is investigated. The objective/ecological function is thermodynamically optimized for different cycle temperatures and respective output power/first law efficiency are computed typically for varying operating factors. The major outcomes of the present work are summarized as follows:

- The three performance parameters rises with $\varepsilon_{\mathrm{H}}, \varepsilon_{\mathrm{L}}, \varepsilon_{\mathrm{R}}$, component efficiencies, $\mathrm{C}_{\mathrm{H} 1}, \mathrm{C}_{\mathrm{L}}, \mathrm{C}_{\mathrm{W}}$, coefficients of pressure recovery and fixed temperature pressure drop ratio whereas their value falls down for the fall in $\varepsilon_{\mathrm{H}}$, $\mathrm{C}_{\mathrm{H}}$.

- It is also observed that the impact of efficiency of the turbine is higher on the peak value of ecological function and the respective output power/first law efficiency are also compared to the efficiency of compressor.

- The thermal efficiency of system increases from $18.29 \%$ to $19.86 \%$ whereas power output increases from $61.25 \mathrm{~kW}$ to $68.82 \mathrm{~kW}$ by incorporating CCC in the system.

- The comparison has been done with the conventional power stations i.e., without the influence of isothermal heat addition and it is observed that the system encounters, an enhancement of $12.36 \%$ in output power and $8.58 \%$ in $1^{\text {st }}$ law efficiency of proposed system with the integration of two heat addition modes.

- It is also noticed that the exhaustive investigations and analysis are still required for the efficiency of compressor while designing realistic gas power plants.

- The formulated results of the system possess decreasing pattern of $\varepsilon_{\mathrm{L}}, \varepsilon_{\mathrm{H} 1}, \varepsilon_{\mathrm{H}}$ and heat capacitances as $\mathrm{C}_{\mathrm{W}}$, $\mathrm{C}_{\mathrm{H} 1}$, and $\mathrm{C}_{\mathrm{L}}$ for the executed results near to practical power plant.

The present analysis can further be applied/extended to other thermal energy conversion systems. In order to design an optimal power plant, the optimal values of various input parameters for Brayton heat engine cycle can further be evaluated by applying different evolutionary algorithm techniques viz. NSGA-II, MOEA/D, PSO and TLBO etc.

\section{NOMENCLATURE}

\section{$\mathrm{A}=\operatorname{Area}\left(\mathrm{m}^{2}\right)$}

$\mathrm{C}=$ Heat Capacitance Rate $\left(\mathrm{kWK}^{-1}\right)$

$\mathrm{k}=$ specific heat ratio

$\mathrm{N}=$ Number of heat transfer units

$\mathrm{P}=$ Power output $(\mathrm{kW})$

$\mathrm{Q}=$ Heat transfer rate $(\mathrm{kW})$

$\mathrm{E}=$ Ecolgical Function $(\mathrm{kW})$

$\mathrm{T}=$ Temperature $(\mathrm{K})$

$\mathrm{U}=$ Overall heat transfer Coefficient $\left(\mathrm{kWm}^{-2} \mathrm{~K}^{-1}\right)$

Greek letters:

$\eta=$ Thermal efficiency

$\varepsilon=$ Effectiveness

\section{Subscripts}

$\mathrm{H}=$ isobaric heat source side

$\mathrm{H} 1=$ isothermal heat source side 
$\mathrm{L}=$ heat sink side

$\mathrm{R}=$ regenerator side

$\mathrm{s}=$ reversible adiabatic /ideal

tur $=$ turbine

comp $=$ compressor

$\mathrm{W}=$ working medium

Bhe $=$ Brayton heat engine

\section{REFERENCES}

[1] Angulo-Brown F. An ecological optimization criterion for finite time heat engines. Journal of Applied Physics 1991; 69 (11): 7465-7469.

[2] Yan Z. Comment on ecological optimization criterion for finite time heat engines. Journal of Applied Physics 1993; 73(7): 3583.

[3] Veccguarelli J, Kawall JG, Wallace JS. Analysis of a concept for increasing the efficiency of a Brayton cycle via isothermal heat addition. International Journal of Energy Research 1997; 21(2): 113-127.

[4] Cheng CY, Chen CK. Ecological optimization of an endoreversible Brayton Cycle. Energy Conversion and Management 1998; 39: 33-44.

[5] Cheng CY, Chen CK. Ecological optimization of an irreversible Brayton cycle. Journal Physics D: Applied Physics 1999; 32: 350-357.

[6] Goktun S, Yavuz H. Thermal efficiency of a regenerative Brayton cycle with isothermal heat addition. Energy Conversion and Management 1999; 40: 1259-1266.

[7] Erbay LB, Goktun S, Yavuz H. Optimal design of the regenerative gas turbine engine with isothermal heat addition. Applied Energy 2001; 68(3): 249-264.

[8] Kaushik SC, Tyagi SK. Finite Time Thermodynamic analysis of an irreversible regenerative closed cycle Brayton heat engine. International Journal of Solar Energy 2002; 22: 141-151.

[9] Arora R, Kaushik SC, Kumar R. Multi-objective optimization of an irreversible regenerative Brayton cycle using genetic algorithm. In 2015 Int Conf on Futuristic Trends on Computational Analysis and Knowledge Management (ABLAZE), IEEE 2015; 340-346. DOI: 10.1109/ABLAZE.2015.7155017

[10] Ust Y, Safa A, Sahin B. Ecological performance analysis of an endoreversible regenerative Brayton heat engine. Applied Energy 2005; 80(3): 247-260.

[11] Ust Yasin, Sahin B, Kodal A, Akcay IH. Ecological coefficient of performance analysis and optimization of an irreversible regenerative Brayton heat engine. Applied Energy 2006; 83: 558-572.

[12] Arora R, Kaushik SC, Kumar R. Multi-objective thermodynamic optimization of solar parabolic dish Stirling heat engine with regenerative losses using NSGA-II and decision making. Applied Solar Energy 2016; 52 (4): 295-304.

[13] Arora R, Kaushik SC, Kumar R, Arora R. Multi-objective thermo-economic optimization of solar parabolic dish Stirling heat engine with regenerative losses using NSGA-II and decision making. International Journal of Electrical Power \& Energy Systems 2016; 74: 25-35.

[14] Arora R, Kaushik SC, Kumar R. Multi-objective optimization of solar powered ericsson cycle using genetic algorithm and fuzzy decision making. In 2015 Int Conf on Advances in Computer Engineering and Applications (ICACEA), IEEE 2015; 553-558. DOI:10.1109/ICACEA.2015.7164754

[15] Arora R, Kaushik SC, Kumar R. Multi-objective thermodynamic optimisation of solar parabolic dish Stirling heat engine using NSGA-II and decision making. International Journal of Renewable Energy Technology 2017; 8 (1): 64-92.

[16] Kaushik SC, Tyagi SK, Singhal MK. Parametric study of an irreversible regenerative Brayton cycle with isothermal heat addition. Energy Conversion and Management 2003; 44: 2013-2025.

[17] Tyagi SK, Kaushik SC, Tiwari V. Ecological optimization and parametric study of an irreversible regenerative modified Brayton cycle with isothermal heat addition, Entropy 2003; 5: 377-390.

[18] Kumar Rajesh, Kaushik SC, Kumar Raj. Performance Analysis of an Irreversible Regenerative Brayton Cycle based on Ecological Optimization Criterion. International Journal of Thermal and Environment Engineering 2015; 9(1): 25-32. 
[19] Tyagi SK, Kaushik SC. Ecological optimization of an irreversible regenerative intercooled Brayton heat engine with direct heat loss. International Journal of Ambient Energy 2005; 26(2): 81-92.

[20] Tyagi SK, Chen J, Kaushik SC. Optimal criterion based on the ecological function of an irreversible intercooled regenerative modified Brayton cycle. International Journal of Exergy 2005, 2(1): 90-107.

[21] Xia D, Chen L, Sun F, Wu C. Universal ecological performance for endoreversible heat engine cycles. International Journal of Ambient Energy 2006; 27(1): 15-20.

[22] Li J, Chen L, Sun F. Ecological performance of an endoreversible Carnot heat engine with complex heat transfer law. International Journal of Thermal Energy 2011; 30: 55-64.

[23] Kumar R, Kaushik SC, Kumar R. Efficient power of Brayton heat engine with friction. International Journal of Engineering Research and Technology 2013, 6 (5): 643-650.

[24] Kumar R, Kaushik SC, Kumar R. Power optimization of an Irreversible regenerative Brayton Cycle using isothermal heat addition. Journal of Thermal Engineering 2015; 1(4): 279-286.

[25] Kaushik SC, Kumar R, Arora R. Thermo-economic optimization and parameteric study of an irreversible Brayton heat engine cycle. Journal of Thermal Engineering 2016; 2 (4): 861-870.

[26] Arora R, Kaushik SC, Kumar R. Performance optimization of Brayton heat engine at maximum efficient power using temp. dependent specific heat of working fluid. Journal of Thermal Engineering 2015; 1 (2): 345-354.

[27] Arora R, Kaushik SC, Kumar Raj, Arora R. Soft computing based multi-objective optimization of Brayton cycle power plant with isothermal heat addition using evolutionary algorithm and decision making. Applied Soft Computing 2016, 46: 267-283.

[28] Kumar R, Kaushik SC, Kumar Raj, Hans R. Multi-objective thermodynamic optimization of irreversible regenerative Brayton cycle using evolutionary algorithm and decision making. Ain Shams Engineering Journal 2016; 7 (2): 741-753.

[29] Razmara M, Bidarvatan M, Shahbakhti M, Robinett RD. Optimal exergy-based control of internal combustion engines. Applied Energy 2016, 183: 1389-1403.

[30] Hajmohammadi MR. Design and analysis of multi-scale annular fins attached to a pin fin. International Journal of Refrigeration 2018; 88 (C): 16-23.

[31] Hajmohammadi MR. Optimal design of tree-shaped inverted fins. International Journal of Heat and Mass Transfer 2018; 116: 1352-1360.

[32] Hajmohammadi MR. Introducing a $\psi$-shaped cavity for cooling a heat generating medium. International Journal of Thermal Sciences 2017; 121: 204-212.

[33] Hajmohammadi MR. Assessment of a lubricant based nanofluid application in a rotary system. Energy Conversion and Management 2017; 146: 78-86.

[34] Arora R, Kaushik SC, Arora R. Multi-objective and multi-parameter optimization of two-stage thermoelectric generator in electrically series and parallel configurations through NSGA-II. Energy 2015; 91: 242-254.

[35] Arora R, Kaushik SC, Arora R. Thermodynamic modeling and multi-objective optimization of two-stage thermoelectric generator in electrically series and parallel configurations. Applied Thermal Engineering 2016; 25 (103): 1312-1323.

[36] Arora R, Arora R. Multiobjective optimization and analytical comparison of single- and 2-stage (series/parallel) thermoelectric heat pumps. International Journal of Energy Research 2018; 42 (4): 17601778. https//doi.org/10.1002/er.3988

[37] Arora R, Arora R. Multicriteria optimization based comprehensive comparative analyses of single- and two-stage (series/parallel) thermoelectric generators including the influence of Thomson effect. Journal of Renewable and Sustainable Energy 2018; 10 (4): 044701.

[38] Arora R, Arora R. Performance Characteristics and Thermodynamic Investigations on Single-Stage Thermoelectric Generator and Heat Pump Systems. Pertanika Journal of Science \& Technology 2018; 26 (4): 1975-1998.

[39] Arora R, Arora R. Experimental Investigations and Exergetic Assessment of $1 \mathrm{~kW}$ Solar PV Plant. Pertanika Journal of Science \& Technology 2018; 26 (4): 1881-1897.

[40] Arora R, Arora R, Sridhara SN. Performance assessment of $186 \mathrm{kWp}$ grid interactive solar photovoltaic plant in Northern India. International Journal of Ambient Energy 2019; 1-28. DOI:

$10.1080 / 01430750.2019 .1630312$ 
[41] Ahmed SU, Arora R. Quality characteristics optimization in CNC end milling of A36 K02600 using Taguchi's approach coupled with artificial neural network and genetic algorithm. International Journal of System Assurance Engineering and Management 2019; 10(4):676-95.

[42] Chiteka K, Arora R, Jain V. CFD Prediction of dust deposition and installation parametric optimisation for soiling mitigation in non-tracking solar PV modules. International Journal of Ambient Energy 201 9; 5:1-14. https://doi.org/10.1080/01430750.2019.1594373

[43] Mohanty S, Arora R, Parkash O. Performance prediction and comparative analysis for a designed, dev eloped, and modeled counterflow heat exchanger using computational fluid dynamics. Computational Thermal Sciences: An International Journal 2019;11(5): 423-443.

[44] Maputi ES, Arora R. Multi-objective spur gear design using teaching learning-based optimization and decision-making techniques. Cogent Engineering 2019; 6(1):1665396.

[45] Chiteka K, Sridhara SN, Arora R. Numerical investigation of installation and environmental parameters on soiling of roof-mounted solar photovoltaic array. Cogent Engineering 2019;6(1):1649007

[46] Chiteka K, Arora R, Sridhara SN. A method to predict solar photovoltaic soiling using artificial neural networks and multiple linear regression models. Energy Systems 2019; 1-22. https://doi.org/10.1007/s12667-019-00348-w

[47] Arora R, Arora R. Parametric Investigations and Thermodynamic Optimization of Regenerative Brayton Heat Engine. In Advances in Fluid and Thermal Engineering 2019; pp. 753-762. Springer, Singapore

[48] Maputi ES, Arora R. Design optimization of a three-stage transmission using advanced optimization techniques. International Journal for Simulation and Multidisciplinary Design Optimization 2019;10: A8.

\section{APPENDIX}

The different parameters are given as below:

$$
\begin{aligned}
& X_{1}=x_{1} x_{3}-x_{2} x_{4} \\
& Y_{1}=x_{12}\left(x_{5}^{2}-4 X y_{5}\right) \\
& x_{1}=\varepsilon_{R}(1-y)\left(1-\eta_{\text {comp }}\right)+\eta_{c o m p} \\
& x_{2}=(1-x)\left(1-\varepsilon_{R}\right) \\
& x_{3}=\beta(1-x)\left(1-\eta_{\text {turb }}^{-1}\right)\left(1-\varepsilon_{R}\right) \\
& x_{4}=(1-y) \varepsilon_{R} \\
& x_{5}=x_{1} y_{3}+x_{3} y_{1}-x_{4} y_{2}-x_{2} y_{4} \\
& x_{6}=x_{1} z_{3}+x_{3} z_{1}-x_{4} z_{2}-x_{2} z_{4} \\
& x_{7}=C_{W}\left\{x\left(1-\varepsilon_{R}\right)+y \varepsilon_{R}+z(1-x)\left(1-\varepsilon_{R}\right)\right\} \\
& x_{8}=C_{W}\left(1-\varepsilon_{R}\right)\{x+z(1-x)\} \\
& x_{9}=C_{W} T_{0}\left(x\left(1-\varepsilon_{R}\right) / T_{H 1}+y \varepsilon_{R} / T_{L 1}+z x_{2} / T_{H 3}\right) x_{10}=x_{7}+x_{9} \\
& x_{11}=X y_{10}^{2} / x_{10}^{2}-x_{5} y_{10} / x_{10} \\
& x_{12}=x_{11}+y_{5} \\
& X_{2}=x_{5} T_{5}+x_{6} \\
& Y_{2}=2 x_{12}\left(x_{5} x_{6}-2 X y_{6}\right) \\
& y_{1}=\left(1-\varepsilon_{R}\right)(1-y)\left(1-\eta_{c o m p}\right) \\
& y_{2}=(1-x) \varepsilon_{R} \\
& y_{3}=\beta\left\{\eta_{t}^{-1}+(1-a)\left(1-\eta_{t}^{-1}\right) \varepsilon_{R}\right\} \\
& y_{4}=\left(1-\varepsilon_{R}\right)(1-y)
\end{aligned}
$$


Journal of Thermal Engineering, Research Article, Vol. 6, No. 1, pp. 28-42, January, 2020

$$
\begin{aligned}
& y_{5}=y_{1} y_{3}-y_{2} y_{4} \\
& y_{6}=y_{1} z_{3}+z_{1} y_{3}-z_{2} y_{4}-y_{2} z_{4} \\
& y_{7}=C_{W}\left\{y\left(1-\varepsilon_{R}\right)+x \varepsilon_{R}+z(1-x) \varepsilon_{R}\right\} \\
& y_{8}=C_{W} \varepsilon_{R}(z(1-x)+x) \\
& y_{9}=C_{W} T_{0}\left(x \varepsilon_{R} / T_{H 1}+y\left(1-\varepsilon_{R}\right) / T_{L 1}+z y_{2} / T_{H 3}\right) y_{10}=y_{7}+y_{9} \\
& X_{3}=y_{5} T_{5}^{2}+y_{6} T_{5}+z_{5} \\
& Y_{3}=x_{11}\left(x_{6}^{2}-4 X z_{5}\right)+y_{6}\left(x_{5} x_{6}-X y_{6}\right)-x_{5}^{2} z_{5} \\
& z_{1}=\left(1-\eta_{c o m p}\right) y T_{L 1} \\
& z_{2}=a T_{H 1} \\
& z_{3}=\beta x\left(1-\eta_{t u r b}^{-1}\right) T_{H 1} \\
& z_{4}=y T_{L 1} \\
& z_{5}=z_{1} z_{3}-z_{2} z_{4} \\
& z_{6}=C_{W}\left(x T_{H 1}+y T_{L 1}+z T_{H 3}-x z T_{H 1}\right) \\
& z_{7}=C_{W}\left(x T_{H 1}(1-z)+z T_{H 3}\right) \\
& z_{8}=C_{W} T_{0}\left(x+y+z-z z_{2} / T_{H 3}\right) \\
& z_{9}=z_{6}+z_{8} \\
& x=\frac{C_{H} \varepsilon_{H}}{C_{W}}, z=\frac{C_{H 1} \varepsilon_{H 1}}{C_{W}}, y=\frac{C_{L} \varepsilon_{L}}{C_{W}} \\
& x_{W}
\end{aligned}
$$

